\title{
DIGITAL MAGNETIC QUANTUM WELLS FOR THE STUDY OF INTERFACE SHARPNESS OF MOLECULAR BEAM EPITAXY GROWN STRUCTURES*
}

T. Wojtowicz, G. Karczewski, A. ZaKrzewski, M. Kutrowski, E. JaniK, E. Dynowska, K. Kopalko, S. Kret, J. Kossut

Institute of Physics, Polish Academy of Sciences

Al. Lotników 32/46, 02-668 Warszawa, Poland

AND J.Y. LAVAL

Laboratoire de Physique du Solide, UPR A0005 CNRS

Ecole Superieure de Physique et de Chimie Industrielles de la Ville de Paris 10, rue Vaquelin, 75231 Paris Cedex 05, France

We report on the growth and basic characterization of digital magnetic quantum wells, that is, quantum wells in which the well material is itself a short period superlattice composed of alternating diluted magnetic and nonmagnetic semiconductor layers each only a few monolayers thick. These novel structures can be useful in a variety of studies, including studies of barrier-well interface sharpness.

PACS numbers: 73.20.Dx,78.55.Et

One of the most important problems in the molecular beam epitaxy (MBE) growth of low-dimensional structures is the problem of sharpness of interfaces between different materials constituting these structures. Diluted magnetic semiconductors (DMS) have already been proposed as a class of materials useful for studying the abruptness of interfaces. The method takes advantage of an enhanced spin-splitting occurring for states in a nonmagnetic quantum well that has a non-abrupt interface with a DMS barrier [1,2]. However, even for an ideal interface the enhancement of spin-splitting can be due to "surface magnetism" related to a decreased number of magnetic neighbors of magnetic barrier atoms at interface $[3,4]$.

In this paper we propose new structures that could help to distinguish between these two effects. Instead of studying regular $\mathrm{QW}$ with two nonmagnetic-magnetic interfaces, we suggest to use digital magnetic quantum wells (DMQW), that

*This work is supported in part by the State Committee for Scientific Research (Republic of Poland) through grant PBZ-ZO11/P4/93/01. 
is, QW in which the well material is itself a short period superlattice (SPSL) composed of alternating DMS and nonmagnetic layers, each only a few monolayers (ML) thick. For such structures not only the number of the interfaces is increased, but also some of these interfaces are located inside the QW where the amplitude of conduction electron and/or valence band hole wave function is large. This should lead to a greatly enhanced sensitivity of the spin-splitting to the non-abrupt nature of the interface and/or to "surface magnetism". Also, for the same reason, DMQWs can be useful for the studies of e.g. decoration of interfaces with impurities, lattice defects, etc.

We report the first successful growth of such $\mathrm{CdTe} / \mathrm{Cd}_{1-x} \mathrm{Mn}_{x} \mathrm{Te}$ digital magnetic quantum wells, and their basic characterization by means of X-ray diffractometry, photoluminescence (PL), and high resolution transmission electron microscopy (TEM).

The set of different multiple DMQW structures was grown by the standard molecular beam epitaxy, using EPI $620 \mathrm{MBE}$ system. Japan Energy Corporation $7 \mathrm{~N} \mathrm{Cd}, \mathrm{Te}, \mathrm{CdTe}$ and $6 \mathrm{~N} \mathrm{Zn}$ as well as Sumitomo (100) GaAs (2 ${ }^{\circ}$-off) epi-ready substrates were used. High purity Mn source material was obtained in the Institute of Physics in Warsaw.

Prior to the growth of quantum wells, we deposited several buffer layers: $\mathrm{ZnTe}(\approx 1000 \AA), \mathrm{CdTe}(\approx 2 \mu \mathrm{m})$, followed by $\mathrm{Cd}_{1-x} \mathrm{Mn}_{x}$ Te buffer $(0.35 \mu \mathrm{m}$ for sample 10183 and $0.1 \mu \mathrm{m}$ for samples 10193 and 10203) with $x$ equal to that in the barriers of the structures subsequently grown on top of these buffers. The growth of the buffer layers was performed at temperatures that were $50^{\circ} \mathrm{C}$ higher than those maintained during the growth of the quantum wells and the barriers. The structural quality of the buffers was checked by X-ray diffraction. The double crystal rocking curve widths of CdTe buffers were 300-400 arcsec.

Each structure consisted of one regular CdTe QW and of several DMQWs with the same well width, that differred, however, in the type of short period superlattice constituting these wells. For example, structure 10183 consisted of four QWs with $87.5 \AA$ well width $(27 \mathrm{ML})$ : one regular CdTe QW and three DMQWs (see Fig. 1). SPSL for the first (in the growth direction) DMQW was composed of three monolayers of CdTe alternated with a single monolayer of $\mathrm{Cd}_{1-x} \mathrm{Mn}_{x} \mathrm{Te}\left(\mathrm{CdTe}[3 \mathrm{ML}] \times \mathrm{Cd}_{1-x} \mathrm{Mn}_{x} \mathrm{Te}[1 \mathrm{ML}]\right.$, denoted by $\left.(3 \times 1)\right)$, for the second well, $2 \mathrm{ML}$ of CdTe alternated with $1 \mathrm{ML}$ of $\mathrm{Cd}_{1-x} \mathrm{Mn}_{x} \mathrm{Te}$ (CdTe[2 ML]× $\mathrm{Cd}_{1-x} \mathrm{Mn}_{x} \mathrm{Te}[1 \mathrm{ML}]$, denoted by $\left.(2 \times 1)\right)$, and for the third well, of a single monolayer of CdTe alternated with a single monolayer of $\mathrm{Cd}_{1-x} \mathrm{Mn}_{x} \mathrm{Te}(\mathrm{CdTe}[1 \mathrm{ML}] \times$ $\mathrm{Cd}_{1-x} \mathrm{Mn}_{x} \mathrm{Te}[1 \mathrm{ML}]$, denoted by $\left.(1 \times 1)\right)$. The wells were separated by $500 \AA$ thick barriers, and the entire structure was completed by $500 \AA$ cap layer. Prior to the growth of QW structures, the growth rate (typically $2.2 \AA / \mathrm{s}$ ) was precisely calibrated via measurements of RHEED oscillations. The growth of the structures was performed without any growth interruptions at relatively low temperatures of about $240^{\circ} \mathrm{C}$.

The luminescence at $10 \mathrm{~K}$ excited by argon-laser beam $(488 \mathrm{~nm})$ in three multiple DMQW structures and in one regular multiple QW structure consisting of eight isolated CdTe wells is presented in Fig. 1. One can clearly see that the widths of the photoluminescence lines of DMQWs is comparable to that in stan- 


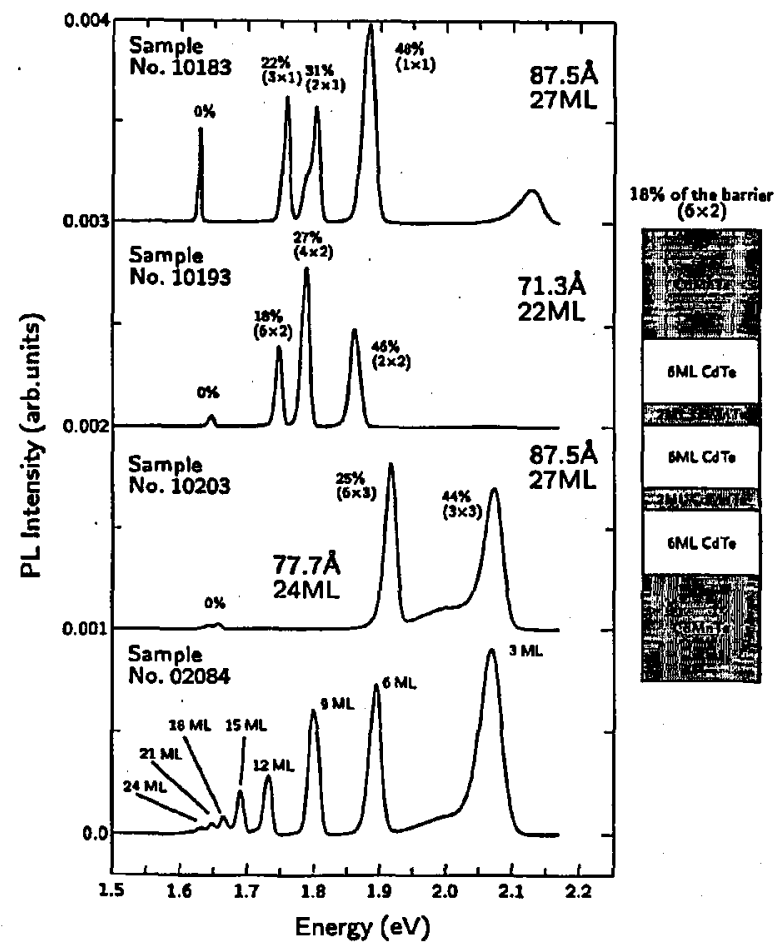

Fig. 1. Photoluminescence spectra for three multiple $\mathrm{CdTe} / \mathrm{Cd}_{1-x} \mathrm{Mn}_{x} \mathrm{Te} \mathrm{DMQW}$ structures excited by $488 \mathrm{~nm}$ argon-laser beam. Photoluminescence of one regular eight-QW structure is plotted for comparison. All data were taken at $T=10 \mathrm{~K}$. The right hand side of the figure shows a schematic picture of the $(6 \times 2)$ DMQW. Structure 10203 has two 24 and one $27 \mathrm{ML}(3 \times 3) \mathrm{QW}$.

dard QWs (lowest spectrum), giving good prospects for a successful application of these DMQWs for magneto-optical studies, including magnetoreflection and magneto-optical Kerr effect.

Since for the short period superlattice one expects the band structure to be very similar to that of a suitable mixed crystal [5], we calculated the energy position of $\mathrm{HH} 1 \rightarrow \mathrm{E} 1$ transition in DMQWs assuming the composition in the well (i.e., the $\mathrm{Mn}$ mole fraction) equal to the ratio of the number of magnetic monolayers in the well to the total number of monolayers in the well, multiplied by the real composition of the barrier. For each DMQW the composition of the well is given in Fig. 1 as a percent of $\mathrm{Mn}$ composition in the barrier. For example, for the sample 10183 the composition of $\mathrm{Mn}$ in the barrier is estimated as $x=$ 0.33 based on the energy position $(2.128 \mathrm{eV})$ of the PL peak from the barrier. Therefore $22 \%$ for $(3 \times 1)$ well means that the real composition of $\mathrm{Mn}$ in the well $x$ is 0.073 . We observed quite good agreement of the calculated energies with the observed energy positions of PL peaks for samples 10183 and 10293. This agreement would be, however, natural if the magnetic layers inside the wells were completely smeared out. Therefore, we performed also a study of the sample 10193 
by means of high resolution transmission electron microscopy. In spite of difficulties in specimen preparation, at least for one DMQW, $22 \mathrm{ML}(6 \times 2)$, the TEM picture shows separate $\mathrm{CdTe}$ and $\mathrm{CdMnTe}$ thin layers within the well region. This strongly confirms that the assumed DMQWs were, in fact, produced by our MBE growth.

In the case of sample 10203 a large blue shift of $(6 \times 3)$ and $(3 \times 3)$ DMQW PL peak positions can be seen in Fig. 1. This could be caused by an unexpected increase in Mn composition in the barrier, in spite of the fact that all our samples were grown under similar flux conditions. Unfortunately, this hypothesis could not be verified by X-rays due to a rather poor crystallographic quality of the thin $\mathrm{CdMnTe}$ barrier in this sample. We would like to point out, however, the very good energy coincidence of the PL peak position of $(3 \times 3)$ DMQW and 3 ML single QW, visible in Fig. 1. Also, the temperature dependence of PL peak position for $(6 \times 3)$ and $(3 \times 3)$ DMQWs is very similar to that of 6 and 3 ML single QW grown with very similar flux conditions as for DMQW structures. Therefore, it is tempting to conclude that barriers as thin as $3 \mathrm{ML}$ can, to a certain degree, localize carriers in the thin well area. Definitely, however, more experiments (especially in the presence of a magnetic field) are required to understand the observed behavior.

In conclusion, we proposed and successfully grew novel DMQW structures that can be used for optimization of MBE growth from the point of view of interface sharpness. Furthermore, high quality structures of this type can be very useful in studies of low-dimensional magnetism. For example, DMQWs with SPSL comprised one ML of cubic MnTe alternated with $n$ MLs of CdTe grown on a (100) plane, and immersed in MnTe barriers, provides a system suitable for studying 2D antiferromagnetism via optical methods. Also, with an increase in $n$, i.e. with increasing separation between magnetic layers, one can try to study the crossover from $3 \mathrm{D}$ to $2 \mathrm{D}$ magnetism, and to obtain information on the range of antiferromagnetic coupling between magnetic ions. The advantage now is that the 2D magnetic material is inside the well and that only several MLs are sufficient to make a useful structure. This not only saves effusion cell shutter lifetime, but also allows one to grow structures of better quality and reproducibility than in the case with thick structures such as these required for SQUID magnetization measurements.

The authors are grateful to Prof. A. Mycielski for supplying high purity Mn used as a source material.

\section{References}

[1] J.A. Gaj, C. Bodin-Deshayes, P. Peyla, J. Cibert, G. Feulliet, Y. Merle d'Aubigné, R. Romestain, A. Wasiela, in: Proc. 21st Int. Conf. Phys. Semicond., Eds. Ping Jiang, Hou-Zhi Zheng, World Scientific, Singapore 1992, p. 1936.

[2] J.A. Gaj, W. Grieshaber, C. Bodin-Deshayes, J. Cibert, G. Feulliet, Y. Merle d'Aubigné, A. Wasiela, submitted to Phys. Rev. $B$.

[3] D.R. Yakovlev, in: Festkörperprobleme / Advances in Solid State Physics, Vol. 32, Ed. U. Rössler, Vieweg, Braunschweig/Wiesbaden 1992, p. 251.

[4] W.J. Ossau, B. Kuhn-Heinrich, Physica B 184, 422 (1993).

[5] J.N. Schulman, T.C. McGill, Appl. Phys. Lett. 34, 663 (1979). 